\title{
Protein-Carbohydrate Interaction
}

\section{Agar Gel-Diffusion Studies on the Interaction of Concanavalin A, a Lectin Isolated from Jack Bean, with Polysaccharides ${ }^{1}$}

\author{
I. J. GOLDSTEIN² AND LUCY L. SO \\ Department of Biochemistry, School of Medicine, State University of New York at Buffalo, Buffalo, New \\ York, and Department of Biological Chemistry The University of Michigan, Ann Arbor, Michigan ${ }^{3}$
}

Received March 31, 1965

\begin{abstract}
Concanavalin A, a protein isolated from the jack bcan (Canavalia ensiformis), has previously been shown to form a precipitate with glycogen and yeast mannan, and more recently with dextrans and amylopectin. Double-diffusion precipitation studies in agar gel have been found to afford a very sensitive and satisfactory method for investigating the interaction of concanavalin A with certain types of branched, glucose- and mannose- containing polysaccharides. In this manner concanavalin $\mathrm{A}$ can be used as a reagent for the detection and preliminary characterization of various types of polysaccharides. Bacterial levans as well as certain plant fructans also form precipitation bands with concanavalin $A$. This observation represents the first report of such an interaction. The method may also provide an indication as to the heterogeneity of polysaccharide preparations that react with concanavalin $\mathbf{A}$ as well as affording preliminary information on the relative molecular size of a series of reactive polysaccharides. Inhibition of the precipitation bands by low molecular weight carbohydrate molecules may be demonstrated. These inhibition studies reveal the highly specific nature of the combining sites of the concanavalin A molecule.
\end{abstract}

Gel diffusion has been a very valuable tool in immunological analysis since its discovery by Bechold (1). In recent years this technique has been extensively developed by Oudin (2), Ouchterlony $(3,4)$, and others $(5,6)$. Utilization of the agar gel-diffusion technique for the investigation of phytohemagglutinins was first reported by Bird $(7,8)$ in his study of blood group substances with precipitins of plant origin (Dolichos biflorus and Ricinus communis).

The study of phytohemagglutinins (commonly called lectins) was initiated in $\mathbf{1 8 8 8}$ with Stillmark's discovery (9) of the presence of a hemagglutinin in castor beans (Ricinus

1 A report of this work was presented at the 149th Meeting of the American Chemical Society, Detroit, Michigan, April, 1965.

${ }^{2}$ Established Investigator of the American Heart Association.

3 Present address. communis). Since then thousands of plant proteins have been examined for their capacity to agglutinate red blood cells (10-12). Among the lectins may be cited concanavalin A (13), a globulin isolated from the jack bean (Canavalia ensiformis). In addition to its agglutinating activity, concanavalin $\mathbf{A}$ was reported by Sumner and Howell (14). to form a precipitate with glycogen and yeast mannan.

Using a turbidimetric assay, Cifonelli et al. (15) employed this protein in the form of crude extracts for the quantitative determination of these polysaccharides. Manners and Wright (16) confirmed and extended these results in an investigation of glycogen from a number of different organisms. More recently, a large number of polysaccharides has been tested for their reactivity with a partially purified preparation of concanavalin $A(17,18)$. Contrary to previous 
reports, $(15,16)$ it was demonstrated that amylopectin also reacts to form a precipitate with concanavalin A. Dextrans were also shown to be capable of reaction, and a large number of these polysaccharides has been examined for their reactivity with the protein. During the past few years certain serum proteins were also reported to react with crude extracts of the jack bean (19-21).

The binding of mono- and oligosaccharides to concanavalin A has been studied by examining the extent to which these small molecules inhibit the concanavalin A-polysaccharide precipitation reactions $(17,22)$. Addition of small molecules that can compete effectively for the combining sites on the protein molecule results in a partial or complete dissolution of the concanavalin A-polysaccharide precipitate. In this manner the stereochemical nature of the combining site of the concanavalin A molecule has been studied.

This paper describes the application of the agar gel double-diffusion technique to the study of polysaccharide-concanavalin A interaction. It will be demonstrated that this technique may serve as a useful tool for the qualitative identification and preliminary characterization of certain types of polysaccharides.

\section{MATERIALS AND METHODS}

Concanavalin A was isolated in a partially purified form from jack bean meal according to the procedure of Sumner and Howell (13). The routine test solution contained $300 \mathrm{mg}$ protein and 10.0 mmoles acetate buffer $(\mathrm{pH} 5.30)$ in $100 \mathrm{ml}$ of half-saturated aqueous sodium chloride.

Plates suitable for agar gel-diffusion studies were prepared (23) as follows: The medium was made by heating to $100^{\circ}$ a $1 \%$ solution of Noble agar (Difco Laboratories, Detroit, Michigan) in $0.85 \%$ saline, buffered with $0.1 \mathrm{M}$ phosphate $(\mathrm{pH}$ $7.2)$. Sodium azide $(1 \%)(5)$ was added as a preservative. A solution of warm agar (approximately $1 \mathrm{ml}$ ) was poured onto the bottom of the plate (Petri dish, box type, $50 \times 12 \mathrm{~mm}$ ) to form a thin layer and allowed to gel. Wells $(0.65 \mathrm{~cm}$ in diameter and $1 \mathrm{~cm}$ distance between well centers), with a capacity of approximately $0.12 \mathrm{ml}$, were formed by means of stainless steel cylinders $(0.65$ $\mathrm{cm}$ outside diameter and $0.70 \mathrm{~cm}$ in length) by pouring a second layer of agar (approximately 4 ml) into the Petri dish. The agar was allowed to gel and the templates were withdrawn with forceps.

In performing diffusion experiments, the concanavalin A preparation was added to the center well and the test solutions $(0.10 \mathrm{ml})$ were placed in the peripheral wells. Plates were incubated at room temperature $\left(20-25^{\circ}\right)$ and observed twice daily. Precipitation bands, which usually commenced forming between 12 and 24 hours, were readily visible. Light green, $p$-phenylenediamine, and amidoschwarz were used as stains for further identification of the precipitation bands and for permanent preservation of the plates. Staining was performed as follows: Excess reactants were removed by washing with saline (containing $0.1 \mathrm{M}$ phosphate buffer, $\mathrm{pH}$ 7.2) for approximately 24 hours. The agar was carefully rimmed off the plate and allowed to dry on a glass slide for 24 hours at room temperature. The dried disc was immersed in a solution of the dye (duration, $5-10$ minutes), and excess dye was washed off with a solution of methanol-acetic acid-water $\langle 5: 1: 5$, $\mathrm{v} / \mathrm{v}$ ). Glycogen and amylopectin were identified by allowing the plates to stand for 10 minutes in a tank saturated with iodine vapor.

For inhibition studies and those relating to the resolution of mixtures, wells were filled with a solution containing inhibitor and polysaccharide in a 1:1 mixture (approximately $0.05 \mathrm{ml}$ of each solution).

Tests for specific interaction were also performed after precipitate formation in the gel had occurred. In this case, a solution of inhibitor (methyl $\alpha$-D-mannopyranoside or melibiose, 10 $\mathrm{mg}$ per milliliter) was layered over the surface of the agar gel, and the time required for the disappearance of precipitate bands was noted. Unless otherwise noted, polysaccharide solutions contained $1 \mathrm{mg}$ per milliliter.

A solution of $\alpha$-amylase suitable for digesting coneanavalin A-glycogen precipitation bands was prepared by dissolving the enzyme (General Biochemicals) in $0.1 \mathrm{M}$ phosphate buffer ( $\mathrm{pH} \mathrm{6.9)}$ which was $0.006 M$ with respect to $\mathrm{NaCl}$. Final concentration of $\alpha$-amylase was $50 \mu \mathrm{g}$ per milliliter. The solution was layered over the entire surface of the agar. It required approximately 2 days for the glycogen-concanavalin A precipitation band to disappear.

Hydrolysis of levan preparations was conducted in $1 \mathrm{~N}$ sulfuric acid by heating on a boiling water bath for 3 hours. The hydrolyzate was neutralized $\left(\mathrm{BaCO}_{3}\right)$, filtered, and concentrated in vacuo at $40^{\circ}$. Paper chromatograms were run with 1 butanol-ethanol-water $(4: 1: 5, v / v)$ as the irrigating solvent and the alkaline silver nitrate spray reagent. 


\section{RESULTS}

Immunodiffusion techniques were used to study the interaction between concanavalin $A$ and various polysaccharides. Figure 1 shows the reaction of concanavalin $A$ with several different types of polysaccharides. Among the carbohydrate polymers forming a precipitation band with concanvalin $\mathrm{A}$ are dextrans, amylopectins, glycogens, yeast mannan, and a chemically synthesized mannan. The structural features common to these polysaccharides will be discussed below. In addition, it was found that several bacterial levans as well as a glucofructan from the Hawaiian $t i$ plant (24) also form precipitation bands with concanavalin A (Fig. 2). This is the first instance of such interaction to be reported.

Table I lists a number of the polysaccharides which were tested for their capacity to form a precipitation band with concanavalin A. Not all of the dextrans tested reacted with the protein (3 $\mathrm{ng}$ per milliliter). Increasing the concentration of concanavalin A (e.g., $25 \mathrm{mg}$ per milliliter), however, showed that all dextrans form precipitation bands (18). The remaining, unreactive poly-

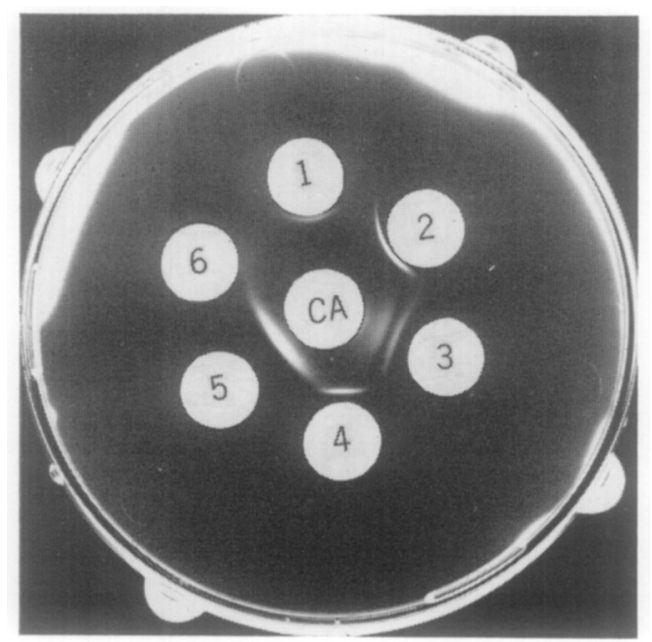

FIG. 1. Agar gel-diffusion pattern. Center well: concanavalin A. Peripheral wells: (1) potato amylopectin, $100 \gamma$; (2) dextran B-1355-S, $100 \gamma$; (3) levan B-1662, $100 \gamma$; (4) rat liver glycogen, $100 \gamma$; (5) synthetic mannan, $20 \gamma$; (6) saline buffered with $0.1 M$ phosphate, $\mathrm{pH} 7.2$.

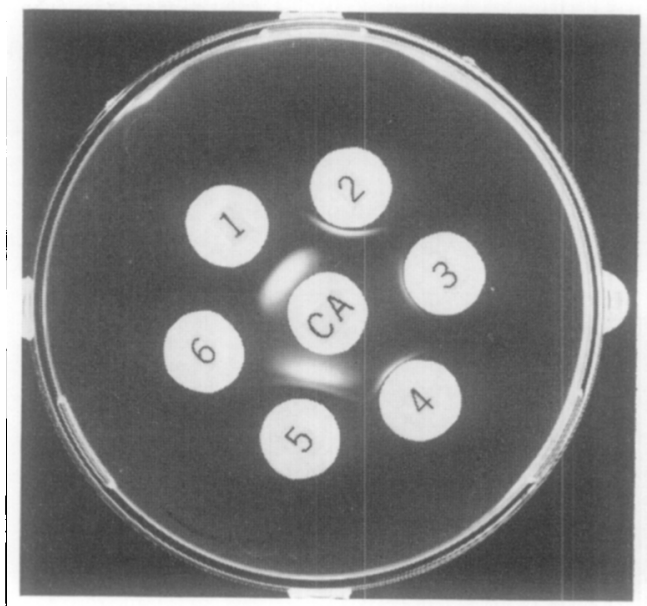

FIg. 2. Agar gel-diffusion pattern. Center well: concunavalin A. Peripheral wells: (1) $T i$ inulin, $100 \gamma$; (2) levan from $B$. megaterium, $100 \gamma$; (3) levan from A. levanicum, $100 \gamma$; (4) levan B-512, $100 \gamma ;(5)$ levan B-1662, $100 \gamma ;(6)$ saline buffered with $0.1 M$ phosphate, $\mathrm{pH} 7.2$.

saccharides do not give positive precipitation reactions even at higher levels of concanavalin $\mathrm{A}$.

Alternatively, by increasing the polysaccharide concentration (10 $\mathrm{mg}$ per milliliter) it was observed that those dextrans unreactive at low protein concentration would form precipitate bands. Most of the dextrans that show this type of behavior are probably less branched (periodate oxidation data) than those which react at a concentration of $1 \mathrm{mg}$ per milliliter. The less reactive dextrans have relatively fewer combining sites (chain ends) which can effectively bind to concanavalin $\mathrm{A}$ to form a precipitate. Increasing the concentration of polysaccharide increases the number of binding sites and results in band formation.

Under the experimental conditions described, the formation of a single sharp band is assumed to indicate a homogenous polysaccharide preparation (however, see Discussion). Formation of more than one band is taken to signify that the polysaccharide preparation is heterogeneous. For example, levan B-1662 (Fig. 1, Well 3; Fig. 2, Well 5) produced several bands, one of which was quite broad and diffuse. This result is in accord with the findings of Wilham et al. 
TABLE I

Interaction of Polysaccharides with Concanavalin a

\begin{tabular}{|c|c|c|}
\hline \multirow[b]{2}{*}{ Polysaccharide } & \multicolumn{2}{|c|}{$\begin{array}{l}\text { Precipitate formation } \\
\text { with concanavalin A }\end{array}$} \\
\hline & $\begin{array}{l}3 \mathrm{mg} \\
\text { protein } / \mathrm{ml}\end{array}$ & $\begin{array}{l}25 \mathrm{mg} \\
\text { protein/ } \\
\mathrm{ml}\end{array}$ \\
\hline
\end{tabular}

1. Dextran B-512(F) ${ }^{a}$

2. Dextran B-1308

3. Dextran B-1208

4. Dextran B-641

5. Dextran B-1254-S

6. Dextran B-1400

7. Dextran B-1394-S

8. Dextran B-1392

9. Dextran B-1255

10. Dextran B-1502

11. Dextran B-1351

12. Dextran B-1438

13. Dextran B-1389

14. Dextran B-1443

15. Dextran B-1298

16. Dextran B-1355-S

17. Dextran B-742-S

18. Dextran B-1299-S

19. Guinea pig liver glycogen

20. Rabbit liver glycogen

21. Human liver glycogen

22. Rat liver glycogen

23. $E$. coli glycogen

24. R. rubrum glycogen

25. Levan NRRL B-512

26. Levan NRRL B-1662

27. Levan from Erwinia ananas

28. Levan from $B$. megaterium

29. Levan from $A$. levanicum

30. Salep mannan

31. Yeast mannan from $\boldsymbol{S}$. cerevisiae

32. Yeast mannan from $S$. rouxii

33. Chemically synthesized mannan

34. Ivory nut mannan

35. Glucomannan from Eastern Hemlock

36. Xylan

37. Laminarin

38. Inulin

39. Oat gum glucan

40. Glucofructan from Ti plant

41. Yeast glucan

${ }^{a}$ These designations were assigned to individual strains of bacteria and their respective dextrans in the Culture Collection of the Northern Utilization Research Branch of the U.S. Department of Agriculture (32).
(25) that levan preparations often contain small amounts of dextran as an impurity. The slower moving component seen as a faint but sharp line could very well indicate

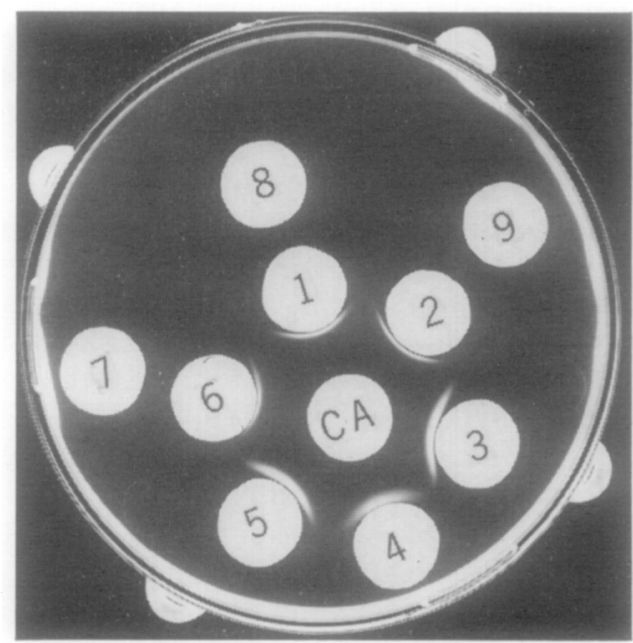

Fta. 3. Agar gel-diffusion pattern. CA: concanavalin A. Peripheral wells: (1), (2), (6) dextran B-1355-s, $100 \gamma$; (3) rat liver glycogen, $100 \gamma$; (4) human liver glycogen, $100 \gamma$, (5) R. rubrum glycogen, $100 \gamma$; (7) egg albumin, $1000 \gamma ;(8)$ bovine serum albumin, $100 \gamma ;$ (9) $\gamma$-globulin.

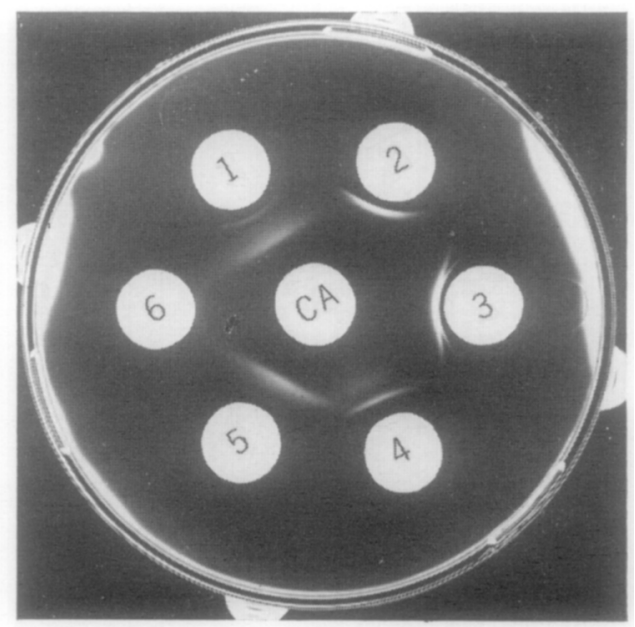

FIG. 4. Agar gel-diffusion pattern. Center well: concunavalin A. Peripheral wells: (1) dextran B-1299, $50 \gamma$ and synthetic mannan, $10 \gamma$; (2) dextran B-1298, $50 \gamma$, and levan B-512, $50 \gamma$; (3) dextran B-1299, $50 \gamma$, and rat liver glycogen, $50 \gamma$; (4) R. rubrum glycogen, $50 \gamma$, and levan B-1662, $50 \gamma$; (5) dextran B-1355-S, $50 \gamma$, and synthetic mannan, $10 \gamma ;(6)$ saline buffered with $0.1 M$ phosphate, pH 7.2. 


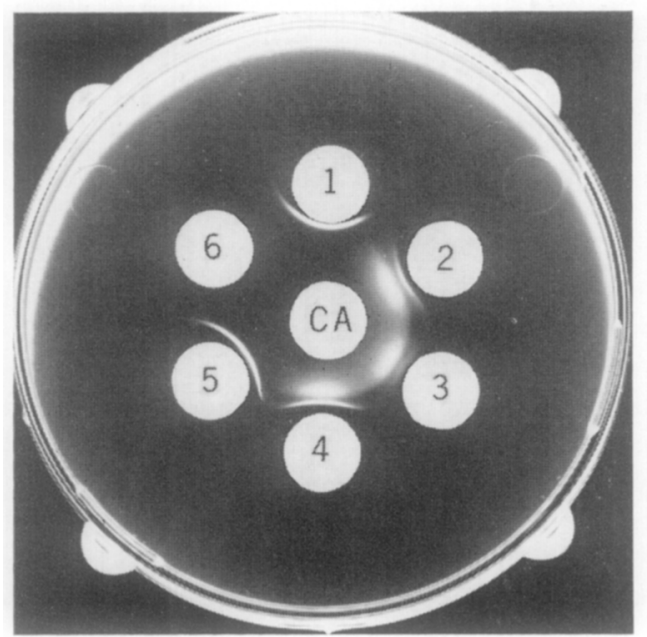

FIG. 5. Agar gel-diffusion pattern. Center well: concanavalin A. Peripheral wells: (1) dextran B-1355-S, $50 \gamma ;(2)$ dextran B-1355-S, $50 \gamma$, and levan B-1662, $50 \gamma$; (3) levan B-1662, $50 \gamma$; (4) levan B-1662, $50 \gamma$, and dextran B-1355-S, $250 \gamma$; (5) dextran B-1355-S, $250 \gamma$; (6) saline buffered with $0.1 M$ phosphate, $\mathrm{pH} 7.2$.

contamination of the levan preparation by a dextran. In support of this hypothesis we noted that the addition of increasing quantities of a dextran to the levan preparation (Fig. 5) increased both the intensity and rate of movement of the slower moving component.

On the other hand, levan B-512 (Fig. 2, Well 4) and those isolated from the growth media of Bacillus megaterium (Fig. 2, Well 2) and A. levanicum (Fig. 2, Well 3) produced only one band, and by this criterion indicate a more homogeneous preparation.

As a control, several other proteins were tested for their reactivity with polysaccharides; Fig. 3 demonstrates the unreactivity of bovine serum albumin. In similar fashion egg albumin and $\gamma$-globulin failed to give precipitates with any polysaccharides.

The number of reactive polysaccharides present in a given mixture may be determined by using direct gel-diffusion studies provided that the polysaccharides of the mixture are capable of forming a visible complex with concanavalin $\mathrm{A}$, and that the diffusion coefficients of the constituent polysaccharides differ to such an extent that precipitation bands for different polysaccharides do not overlap. Figure 4 shows experi- mental evidence for the applicability of this technique as a test for homogeneity. All polysaccharide solutions in the peripheral wells consisted of a $50 \%$ mixture (1:1 by volume) of the constituent polysaccharides. It is apparent from Fig. 4 that when two polysaccharides differ widely in their molecular weights, the best separation was achieved (see Well 1). The two distinct bands formed by the glycogen-dextran mixture (Well 3) were distinguished by the iodine stain. Thus the band corresponding to the glycogen-concanavalin A complex (furthest removed from Well 3) was stained dark brown with iodine, but the slower moving band (dextran) did not react. Further identification of the glycogen component was shown when a solution of $\alpha$-amylase preferentially caused the disappearance of the glycogen-concanavalin $\mathrm{A}$ precipitation band.

From Fig. 5 it can be seen that components of mixtures can tentatively be identified from band position if controls are run simultaneously. Such preliminary identification based on band position requires careful control of polysaccharide concentration. This requirement can be seen from a consideration of Fig. 6, which shows that band position depends on polysaccharide

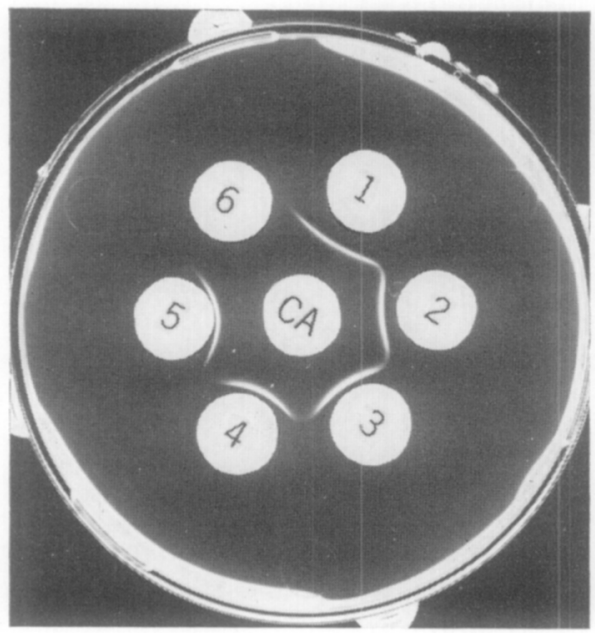

FIG. 6. Agar gel-diffusion pattern. Center well: concanavalin A. Peripheral wells: (1) dextran B-1355-S, $5000 \gamma ;(2)$ dextran B-1355-S, $2500 \gamma$; (3) dextran B-1355-S, $1000 \gamma$; (4) dextran B-1355-S, $500 \gamma ;(5)$ dextran B-1355-S, $100 \gamma ;(6)$ saline buffered with $0.1 M$ phosphate, $\mathrm{pH} 7.2$. 
concentration, the most concentrated polysaccharide solution (Well 1) giving rise to a precipitation band closest to the concanavalin A well.

Since the precipitation bands were stained by a dye characteristically reactive with carbohydrates ( $p$-phenylenediamine), as well as protein dyes (light green and amidoschwarz), the visible white band is evidently a carbohydrate-protein complex.

Inhibition experiments were performed with the following sugars: $\mathrm{D}$ - and L-arabinose, $\mathrm{D}$-xylose, D-glucose, D-mannose, D-galactose, methyl $\alpha$-D-xylopyranoside, methyl $\alpha$-Dmannopyranoside, methyl $\alpha$-D-glucopyranoside, lactose, maltose, melibiose, $\alpha, \alpha$ trehalose, and melezitose. With the exception of D-galactose, lactose, melibiose, and the pentoses, all of the above sugars inhibited the concanavalin $\mathrm{A}$ reaction. Methyl $\alpha$-Dmannopyranoside was the most powerful inhibitor. The minimum amount of methyl $\alpha$-D-mannopyranoside required for $100 \%$ inhibition of the reaction lies between 150 and $200 \mu \mathrm{g}$ for a solution containing approximately $50 \mu \mathrm{g}$ of dextran.

In order to demonstrate further the specific nature of the concanavalin Apolysaccharide interaction, a solution of inhibitor was layered over the developed gel-diffusion plate. In the case of melibiose, a noninhibitor of the concanavalin Apolysaccharide interaction, no change in the appearance of the precipitate bands occurred. On the other hand, when methyl $\alpha$-D-mannopyranoside was employed as the inhibitor, complete disappearance of the precipitate bands occurred, usually within 10-15 minutes. One exception to this observation was the yeast mannan concanavalin A precipitate band. This required several days to disappear, demonstrating we believe, the greater affinity of the concanavalin A receptor sites for $\alpha$-D-mannopyranosyl end units over those of $\alpha$-Dglucopyranosyl termini. This interpretation is in agreement with our observation that D-mannose and its derivatives were invaribly better inhibitors than D-glucose and its derivatives.

\section{DISCUSSION}

The use of immunochemical techniques for studying the fine structure of poly- saccharides has been firmly established (26). Indeed the immunochemical approach may become a routine procedure in laboratories concerned with identification and characterization of carbohydrate polymers. Concanavalin A is a naturally occurring protein which displays many of the properties of an antibody in that it is capable of interacting with a restricted group of polysaccharides to form a precipitate. This analogy to the precipitin reaction between antibody and antigen is further strengthened by its specificity, concentration dependence on both protein and polysaccharide, and capacity to be inhibited specifically by low molecular weight "haptens." In order to differentiate these proteins from immune antibodies, however, Buyd has suggested using the term "lectin" (27).

All previous studies concerned with the precipitation reaction between concanavalin $A$ and polysaccharides have made use of turbidimetric measurements. The present, investigation demonstrates that polysaccharide-concanavalin $\mathrm{A}$ interaction can be studied by means of the agar gel-diffusion technique. In almost all respects this type of study can duplicate turbidimetric measurements with the added advantage that smaller quantities of material are required. Furthermore, a second dimension has been added to the turbidimetric studies reported earlier in that the precipitate may often be resolved into several distinct precipitation bands, thus providing an indication of purity and molecular size distribution.

Multiple precipitation bands for a single polysaccharide have not been observed. Although there are reports of such occurrences, it is generally believed that unless there are sudden variations in temperature, agar gel-diffusion in two dimensions does not suffer from this shortcoming (28).

Control experiments employing other proteins (egg albumin, bovine serum albumin, $\gamma$-globulin, etc.) reveal that these substances do not form precipitation bands with polysaccharides under the experimental conditions described, demonstrating the specificity of the concanavalin A-polysaccharide interaction.

It has been suggested above that double diffusion in agar gel can give information on the homogeneity of polysaccharide 
preparations. Two conditions are necessary for such an evaluation: (1) All components of the mixture must be capable of forming a visible complex with concanavalin $\mathrm{A}$ and must be present in sufficient concentration to be detected. (2) The precipitation bands of the component polysaccharides must not overlap. Synthetic mixtures composed of glycogens, dextrans, and levans were resolved and differentiated on the basis of simultaneously run controls, differential staining, and the preferential dissolution of precipitate bands in the presence of specific enzymes. In this manner we could obtain tentative evidence for the presence of dextran in a levan preparation.

The mobility of a polysaccharide in this system is dependent on its concentration and is a function of its diffusion coefficient, which in turn is related to its molecular weight. Therefore, it would be anticipated that information regarding the molecular weight of polysaccharides reacting with concanavalin A could be obtained. Preliminary studies with dextran, glycogen, levan, and a chemically synthesized mannan suggest that such assessment of molecular weights may be possible. Further studies with preparations of known molecular weight distribution must be conducted before definitive information on the feasibility of this approach can be obtained.

These studies support previous suggestions from this laboratory that polysaccharides which precipitate with concanavalin A must have certain common structural features. Thus, all reactive polysaccharides are ramified and contain terminal, nonreducing $\alpha$-D-ghucopyranosyl or $\alpha$-D-mannopyranosyl units. The dextrans, glycogens, yeast mannans, and amylopectins obviously fall within this category. However, that certain levans and a chemically synthesized mannan form precipitation bands requires comment.

For the first time levans are reported to interact to form a precipitate with concanavalin A. Inulin, another polyfructose polymer, does not react. It is known that bacterial levans (29) are high molecular weight, branched fructans in which $\beta$-Dfructofuranosyl units are linked by $(2 \rightarrow 6)$. glycosidic linkages. Branch points originale from the C-1 hydroxyl group of certain fructofuranosyl units. The occurrence of D-glucose in some levans has been reported. The levan specimens described herein all react with concanavalin $\mathrm{A}$. Hydrolysis with mineral acid followed by paper chromatographic examination reveals that some preparations contain only fructose, whercas others possess variable quantities of glucose. This was especially true of levan preparation B-1662, which contained a considerable quantity of glucose. Its heterogeneity and possible contamination with dextran are demonstrated in Figs. 1 and 2.

The precise structural features which enable levans to react with concanavalin A are currently under investigation. Previous observations $(17,22)$, however, have led to the hypothesis that it is the hydroxyl groups at C-3, C-4, and C-6 of the $\alpha$-Dglucopyranosyl and $\alpha$-D-mannopyranosyl ring forms which bind to the protein. The $\beta$-D-fructofuranosyl residues which occupy chain ends in levans contain hydroxyl groups (at C-3, C-4, C-5, and C-6) of the same configuration, and it may well be these fructofuranosyl units which form the basis for interaction with concanavalin A. Additional evidence that terminal, nonreducing $\beta$-D-fructofuranosyl units bind to concanavalin $A$ is shown by the fact that methyl $\beta$-D-fructofuranoside is a good inhibitor of the polysaccharide-concanavalin $A$ interaction (30). The glucofructan from the Hawaiian $t i$ plant has been shown to contain on the average four terminal nonreducing $\beta$-D-fructofuranosyl residues for every fourteen hexose units (24).

The chemically synthesized mannan (31) is believed to be highly branched and to possess some $\alpha$-D-mannopyranosyl units as nonreducing termini. Preliminary chemical investigation tends to confirm these conclusions and will be reported in detail elsewhere.

A further demonstration of the specificity of the interaction between concanavalin A and polysaccharides is afforded by inhibition studies. As reported above low molecular weight carbohydrate molecules whose configuration is complementary to the binding sites of concanavalin A will occupy these sites and inhibit the formation of precipitalion bands. A report from this laboratory suggested that sugars possessing the $\mathrm{D}$ - 
glucopyranosyl and D- mannopyranosyl configuration were the only good inhibitors of this interaction. $\alpha$-Glycoside formation vastly enhances the capacity of the D-glucose or D-mannose molecule to inhibit concanavalin A-polysaccharide interaction. Inhibition studies with the agar gel-diffusion technique were found to be satisfactory. Of the inhibitors tested, methyl $\alpha$-D-mannopyranoside appears to be one of the most potent. This is in accord with turbidimetric studies conducted in the conventional manner.

\section{ACKNOWLEDGMENTS}

The authors thank Dr. S. Shulman and Miss Larean Hubner for helpful discussions and suggestions, and Miss Susan Kozmycz for photographing the gel-diffusion plates. Mr. Bipin Agrawal prepared most of the concanavalin A used in this study. Many of the levan specimens and all of the dextrans were kindly provided by Dr. Allene Jeanes.

This research was supported by USPHS grants AI-05542 and GM-09364.

\section{REFERENCES}

1. Bechold, H., Z. Phys. Chem. 52, 185 (1905).

2. Oudr, J., in "Methods in Medical Research." (A. C. Corcoran, ed.), Vol. 5, p. 335. Year Book Publishers, Chicago, Illinois (1952).

3. Ouchterlony, Ö., Acta Pathol. Microbiol. Scand. 25, 186 (1948).

4. OUChterlony, Ö., Proc. Intern. Congr. Microbiol., 6th, Rome, 1953. 1, 546 (1953).

5. Feinberg, J. G., Int. Arch. Allergy 11, 129 (1957).

6. WUNDERLY, C., Experientia 13, 421 (1957).

7. BIRD, G. W. G., Vox Sang. 4, 307 (1959).

8. Bird, G. W. G., Vox Sang. 4, 313 (1959).

9. Stillmark, H., "Der giftige Eiweisskorper Ricin: seine Wirkung auf das Blut." Inaugural Dissertation, Dorpat (1888).

10. Morgan, W. T. J., and Watkins, W. M., Brit. J. Exptl. Pathol. 34, 94 (1953).

11. Makela, O., "Studies in Hemagglutinins of Leguminosae Seeds." Academic Dissertation, Helsinki, 1957.

12. Boyd, W. C., Vox Sang. 8, 1 (1963).
13. Sumner, J. B., And Howell, S. F., $J$. Bacteriol. 32, 227 (1936).

14. Sumner, J. B., And Howeld, S. F., J. Biol. Chem. 115, 583 (1936).

15. Cifonelli, J. A., Montgomery, R., AND Smith, F., J. Am. Chem. Soc. 78, 2485 (1956).

16. Manners, D. J., and Wright, A., J. Chem. Soc. 1962, 4592.

17. Goldstein, I. J., Hollerman, C. E., AND Merrick, J. M., Federation Proc. 22, 297 (1963).

18. Goldstein, I. J., Hollerman, C. E., And Merrick, J. M., Biochim. Biophys. Acta 97, 68 (1965).

19. Harris, H., and Robson, E. B., Vox Sang. 8, 348 (1963).

20. Nakamura, S., Tanaka, K., and Murakawa, S., Nature 188, 144 (1960).

21. Murakawa, S., and Nakamura, S., Bull. Yamaguchi Med. School 10, No. 1, 11 (1963).

22. Goldstein, I. J., Hollerman, C. E., AND Sмiтн, E. E., Biochemistry 4, 876 (1965).

23. Barnes, G. W., Soanes, W. A., Mamrod, L., Gonder, M. J., and Shulman, S., J. Lab. Clin. Med. 61, 578 (1963).

24. Boggs, L. A., And Smith, F., $J$. Am. Chem. Soc. 78, $1880(1956)$.

25. Wilham, C. A., Alexander, B. H., and Jeanes, A., Arch. Biochem. Biophys. 59, 61 (1955).

26. HutDelberger, M., in "Fortschritte Der Chemie Organische Naturstaffe" (L. Zechmeister, ed.), Vol. 18, p. 503. Springer, Wren (1960).

27. Boyd, W. C., and Shapleigh, E., Science 119, 419 (1954).

28. Crnwt.e, A. J., "Immunodiffusion," p. 67, Academic Press, New York (1961).

29. BARKER, S. A., in "Comprehensive Biochemistry" (M. Florkin and E. H. Stotz, eds), Vol. 5, p. 252, Elsevier, Amsterdam (1963).

30. So, L. L., unpublished results.

31. Desouza, R., and Goldstein, I. J., unpublished results.

32. Jgangs, A., Haynes, W. C., Wilimam, C. $\Lambda$., Rankin, J. C., Melvin, E. H., Austin, M. J., Cuuskey, J. E., Fisher, B. E., Tsuchiya, H. M., and Risit, C. E., $J . A m$. Chem. Soc. 76, 5041 (1954). 